\title{
Estudio comparativo entre los enfoques de diseño experimental robusto de Taguchi y tradicional en presencia de interacciones de control por control
}

\section{Comparative Study Between the Two Experimental Design Approaches Taguchi and Traditional in Presence of Control by Control Interactions}

\author{
Arias-Nava Elías Heriberto \\ Departamento de Ingeniería Industrial \\ Instituto Tecnológico de Celaya \\ Correo:eariasnava@gmail.com \\ Ríos-Lira Armando Javier \\ Departamento de Ingeniería Industrial \\ Instituto Tecnológico de Celaya \\ Correo:armando.rios@itcelaya.edu.mx
}

\author{
Vázquez-López José Antonio \\ Departamento de Ingeniería Industrial \\ Instituto Tecnológico de Celaya \\ Correo:antonio.vazquez@itcelaya.edu.mx \\ Pérez-González Russell \\ Departamento de Ingeniería Industrial \\ Instituto Tecnológico de Celaya \\ Correo:rupp_hamlet@hotmail.com
}

Información del artículo: recibido: octubre de 2013, aceptado: enero de 2014

\section{Resumen}

El diseño de experimentos desempeña un papel importante dentro del campo de diseño y optimización de procesos y productos, particularmente en áreas de manufactura y mejora continua. Dentro del diseño de experimentos existen múltiples áreas, una de ellas es el diseño robusto. El diseño paramétrico robusto es un principio que enfatiza la correcta selección de niveles de factores de "control", que hacen al producto robusto a la variabilidad introducida por otro grupo de factores llamados factores de "ruido". Este artículo se enfoca en la realización de un estudio comparativo entre dos metodologías conocidas de diseño robusto: el enfoque tradicional y el enfoque de Taguchi, haciendo especial hincapié en el efecto que tienen las interacciones de control por control sobre las condiciones óptimas de operación. Los resultados mostraron que los arreglos cruzados de Taguchi no tienen la capacidad para estimar todos los términos significativos en un modelo. El resultado de las optimizaciones concluye que el enfoque de Taguchi es menos eficiente que el enfoque tradicional, tanto al minimizar como al maximizar.

\section{Descriptores:}

- diseño de experimentos

- diseño robusto

- enfoque de Taguchi

- enfoque tradicional

- arreglo cruzado

- arreglos ortogonales

- resolución mixta 


\begin{abstract}
Design of experiments plays an important role in the field of creating and innovating process and products directly in manufacturing and improving areas. There are several areas into designs of experiments; robust design is one of them. Robust parameter design is a principle that emphasize in products creation through a correct selection of values called "control" which make a product robust to the variability by the noise introducing by another factors known as "noise" factors. This article aims for a comparative study between two well-known robust design methodologies, making a special emphasis in the control by control interaction effects over optimal operating conditions. The results showed that Taguchi's crossed arrays are unable to estimate all significant terms in a model. The optimizations result concludes that the Taguchi's approach is less efficient than the traditional approach in both; maximization and minimization.
\end{abstract}

\section{Introducción}

Existen dos enfoques principales para experimentación robusta: el enfoque de Taguchi y el enfoque tradicional. La metodología de Taguchi para el RPD (diseño paramétrico robusto) fue introducida a principios de los años 80 . Esta metodología gira en torno al uso de diseños ortogonales donde un arreglo ortogonal que involucra variables de control (arreglo interno), se cruza con un arreglo ortogonal que contiene a las variables de ruido (arreglo externo).

En la presente investigación se trabajó con diseños que contienen de 5 a 10 variables, incluidas variables de control y variables de ruido, además de interacciones de control por ruido que hacen a un diseño robusto, y se hizo énfasis en agregar interacciones de control por control significativas al modelo.

La metodología que se utilizó fue en esencia una comparación directa entre los dos enfoques de experimentación a través de indicadores de desempeño, que son estadísticos comúnmente utilizados en la evaluación de diseños de experimentos, donde previamente se colocaron los arreglos cruzados de Taguchi en forma estándar para simplificar su análisis y lograr una comparación directa contra el enfoque tradicional.

Como parte del método se consideró agregar un nivel de ruido medio para los experimentos. Otras variantes dentro del proceso de experimentación fueron el incremento gradual del número de interacciones de control por control significativas en cada modelo, asimismo se incrementó también en forma gradual el nivel de significancia de estas interacciones.

El estudio se basó en caracterizar la influencia de las interacciones de control por control y determinar hasta qué punto marcan una diferencia entre los resultados reportados por ambos enfoques de experimentación robusta, una diferencia encontrada entre las metodolo- gías fue que la correcta estimación de términos en el modelo de regresión lineal no es igual para ambas.

La parte final de la investigación se centró en la búsqueda de condiciones de operación que optimicen la respuesta, esto es esencial para mostrar las diferencias que presentan ambos métodos cuando en un experimento no se estimaron correctamente las interacciones de control por control, que es el caso de la metodología de Taguchi. Estas diferencias se detectaron mediante experimentos que tenían como objetivo maximizar y minimizar la respuesta, partiendo de un modelo verdadero que sirvió de base para simular la información.

El modelo verdadero, se refiere al punto de partida de cada experimento; a la asignación de coeficientes de regresión para efectos principales e interacciones que existirán en el modelo y a la determinación del nivel de ruido y el nivel de significancia de las variables involucradas. A lo largo del análisis de cada experimento el modelo verdadero debe ser el mismo para ambas metodologías. Se le llama verdadero porque constituye la referencia real que se tiene para hacer comparaciones, cuantificar estimaciones y emitir conclusiones acerca del desempeño de cada enfoque experimental.

\section{Desarrollo}

La primera parte de este estudio se basó en una comparación directa entre los enfoques de Taguchi y tradicional, esta comparación se realizó tomando en cuenta una serie de indicadores de desempeño, algunos de estos indicadores incluyen:

- $\mathrm{R}^{2}$ (coeficiente de determinación)

- $\mathrm{R}^{2}$ ajustada

- $\mathrm{R}^{2}$ de la predicción

- Error tipo I (el modelo de regresión tiene términos adicionales) 
- Error tipo II (el modelo de regresión obtenido tiene términos faltantes)

- No error (el modelo de regresión obtenido es el correcto)

Una comparación directa entre los enfoques de Taguchi y tradicional puede resultar difícil, ya que las salidas de software para los dos enfoques son diferentes. El problema se simplifica al darse cuenta de que el arreglo cruzado de Taguchi puede representarse en forma estándar. La figura 1 muestra un arreglo cruzado de Taguchi en forma estándar.

En un arreglo cruzado de Taguchi en forma estándar el arreglo externo se elimina y se integra al arreglo interno, dando como resultado un diseño que puede capturarse en un software estadístico como Minitab o Design Expert para obtener fácilmente los estadísticos mencionados, lo que significa que la comparación entre los dos enfoques es directa. Es importante señalar que a pesar de que la metodología de Taguchi existe desde los 80 , no existe información de tipo cuantitativa sobre las diferencias entre los enfoques de Taguchi y tradicional, la literatura actual solo menciona aspectos cualitativos y muchos libros únicamente mencionan las múltiples críticas hechas a la metodología de Taguchi.

Los modelos verdaderos generados fueron modelos de primer orden que contenían efectos principales de control y ruido, interacciones de control por control e interacciones de control por ruido. El número y el nivel de significancia de las interacciones de control por control se incrementó gradualmente, por ejemplo, si en primera instancia se tenían 3 interacciones de control por control poco significativas en el siguiente paso se incrementó a 4 interacciones de control por control más significativas, posteriormente a 5 y así sucesivamente hasta encontrar diferencias importantes entre los resultados proporcionados por los dos métodos, esto se hizo con el fin de investigar el efecto de estas interacciones de control por control sobre el modelo de regresión lineal reportado por ambas técnicas; de la misma manera se investigó en qué punto estas interacciones comienzan a hacer que el método de Taguchi proporcione resultados erróneos al momento de optimizar el proceso en estudio. Se emplearon simulaciones Monte Carlo para el análisis. La figura 2 ilustra de este proceso.

Las condiciones que debía cumplir el modelo verdadero a partir del que se trabajó son que el número de variables de control debía ser mayor que el número de variables de ruido (VC > VR) y que debía contener al menos una interacción de control por ruido significativa en el modelo. A continuación se muestra un ejemplo:

$y=\beta_{0}+\beta_{1} x_{1}+\beta_{2} x_{2}+\beta_{12} x_{1} x_{2}+x_{1} z_{1}+\phi_{11} x_{1} z_{1}+\phi_{21} x_{2} z_{1}+E$

\section{Condiciones}

- Variables de control: las variables de control se agregaron gradualmente a los modelos verdaderos iniciando con 2 variables y terminado con 8 . Se consideraron significativas la mitad de las variables que se definieron para cada modelo, por ejemplo, para un modelo que contiene 6 variables de control, 3 de ellas serian significativas, para uno que contiene 4 variables de control, 2 de ellas serían significativas, para casos en que el número de variables de control es impar, el número de variables significativas se redondea al entero superior más próximo.

- Variables de ruido: siguen las mismas reglas que las de control, pero condicionadas a que (VC > VR).

- Interacciones de control por control: las interacciones de este tipo estuvieron limitadas a contener los efectos principales que se definieron como significativos dentro de cada modelo verdadero. La cantidad

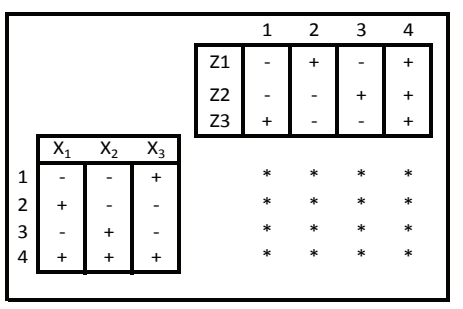

\begin{tabular}{|c|c|c|c|c|c|c|}
\cline { 2 - 7 } \multicolumn{1}{c|}{} & \multicolumn{5}{|c|}{ Factors } \\
\hline 1 & -1 & -1 & 1 & -1 & -1 & 1 \\
2 & -1 & -1 & 1 & 1 & -1 & -1 \\
3 & -1 & -1 & 1 & -1 & 1 & -1 \\
4 & -1 & -1 & 1 & 1 & 1 & 1 \\
5 & 1 & -1 & -1 & -1 & -1 & 1 \\
6 & 1 & -1 & -1 & 1 & -1 & -1 \\
7 & 1 & -1 & -1 & -1 & 1 & -1 \\
8 & 1 & -1 & -1 & 1 & 1 & 1 \\
9 & -1 & 1 & -1 & -1 & -1 & 1 \\
10 & -1 & 1 & -1 & 1 & -1 & -1 \\
11 & -1 & 1 & -1 & -1 & 1 & -1 \\
12 & -1 & 1 & -1 & 1 & 1 & 1 \\
13 & 1 & 1 & 1 & -1 & -1 & 1 \\
14 & 1 & 1 & 1 & 1 & -1 & -1 \\
15 & 1 & 1 & 1 & -1 & 1 & -1 \\
16 & 1 & 1 & 1 & 1 & 1 & 1 \\
\hline
\end{tabular}

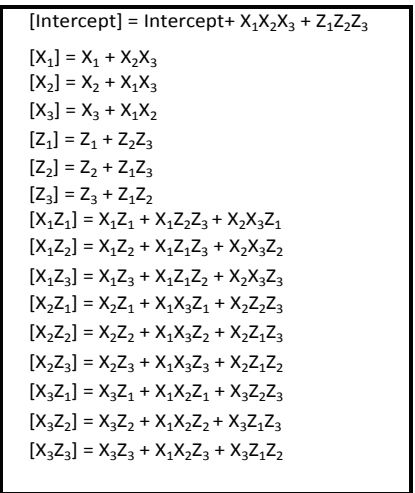

Figura 1. Arreglo cruzado de Taguchi que representa en forma estándar (Ríos et al., 2009) 
de estas interacciones se incrementó gradualmente hasta encontrar una situación en la que uno de los enfoques de experimentación robusta dejara de ser eficiente.

- Interacciones de control por ruido: las interacciones de este tipo son las que hacen que el diseño sea considerado robusto, por lo tanto deben incluirse dentro de cada modelo, se consideraron interacciones significativas aquellas que podían formarse mediante una combinación de factores de control y ruido que resultaron significativos, es decir, si la variable de control A y la variable de ruido G resultaron significativas, una interacción de control por ruido seria $A G$. Estas interacciones no se incrementaron en los pasos subsecuentes al modelo inicial, puesto que solo se establecieron para hacer que el problema sea robusto, pero no son un factor primordial para el análisis.

- Interacciones de ruido por ruido: no se agregaron a ningún modelo por considerarse que no proporcionan información relevante.

\section{Optimización de modelos}

Uno de los objetivos de esta investigación fue comprobar cuál de los dos enfoques de diseño experimental robusto era más eficiente en términos de optimizar la variable de respuesta, para realizar una comparación directa entre ambos métodos, los conjuntos de informa-

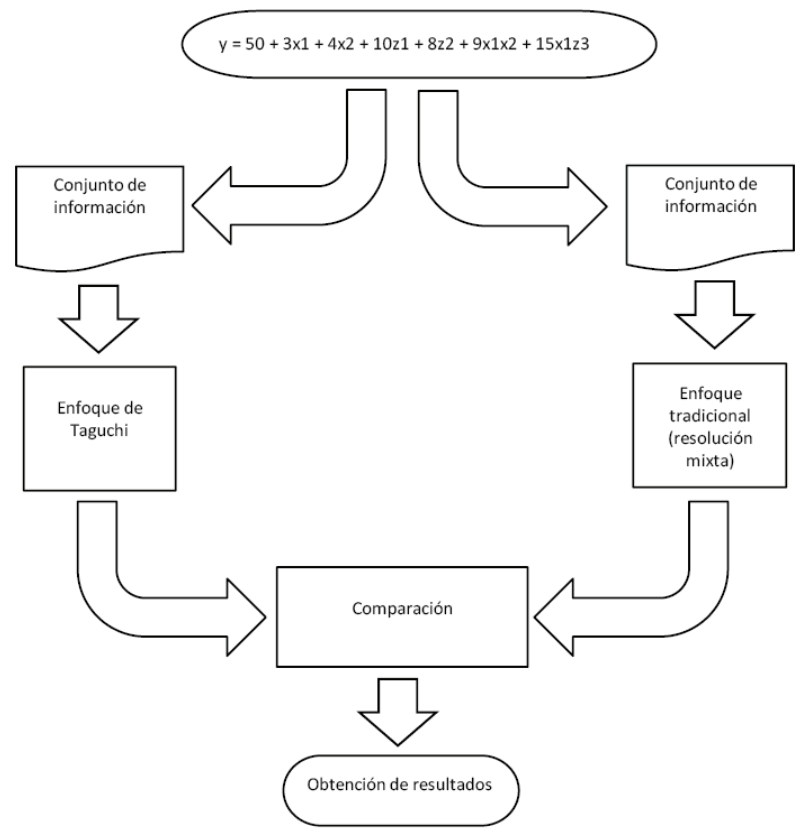

Figura 2. Metodología propuesta para realizar una comparación directa entre los enfoques de Taguchi y tradicional ción simulados se optimizaron para cada metodología y los resultados se compararon utilizando el software Minitab.

Enfoque de Taguchi

Los arreglos cruzados de Taguchi se optimizaron utilizando el software Qualitek-4, el proceso de búsqueda que utiliza este software es el índice de señal a ruido, dependiendo del valor objetivo de la variable de respuesta, mayor es mejor (maximizar) o menor es mejor (minimizar), dicho procedimiento pretende encontrar el valor alto o bajo (entre 1 y -1 ) al que debe colocarse cada variable. Los índices de señal a ruido propuestos por Taguchi se observan en la tabla 1 (Castaño y Domínguez, 2010).

\section{Enfoque de Tradicional}

El enfoque tradicional utiliza la función de deseabilidad y es uno de los métodos más utilizados en la industria para la optimización de procesos de respuesta múltiple. Se basa en la idea de que la "calidad" de un producto o proceso que tiene varias características de calidad, con una de ellas fuera de ciertos límites, es completamente inaceptable. El método encuentra las condiciones de operación $x$ que ofrecen los valores de respuesta más "deseables". La idea básica del enfoque de función de deseabilidad es transformar un problema de respuestas múltiples en un problema de una sola respuesta por medio de transformaciones matemáticas.

La función de deseabilidad se utilizó para la optimización de las variables de respuesta de los conjuntos de información obtenidos mediante el enfoque tradicional de diseño experimental robusto. La función de deseabilidad trabaja buscando valores apropiados que se asignan a cada variable de entrada con el propósito de maximizar un índice de deseabilidad general que varía entre 0 y 1 , el cual es el resultado de la optimización simultanea de varias variables de respuesta.

El software Design Expert basa su optimización numérica en la función de deseabilidad. La deseabilidad

Tabla 1. Índice de señal a ruido

\begin{tabular}{|c|c|c|}
\hline Objetivo & Índice señal a ru & aido \\
\hline Tipo: Menor es mejor & \multirow{3}{*}{$\mathrm{S} / \mathrm{R}_{\mathrm{me}}=-10 \log 10$} & \multirow{3}{*}{$\frac{\sum y i^{2}}{2}$} \\
\hline $\begin{array}{l}\text { Nombre: Respuesta es no negativa y } \\
\text { el valor objetivo ideal es cero. }\end{array}$ & & \\
\hline Tipo: Mayor es mejor & & \\
\hline $\begin{array}{l}\text { Nombre: Respuesta es no negativa } \\
\text { y el valor objetivo ideal infinito es } \\
\text { óptimo. }\end{array}$ & $\mathrm{S} / \mathrm{R}_{\mathrm{ma}}=-10 \log 10$ & $\left(\sum \frac{1}{y i^{2} / n}\right.$ \\
\hline
\end{tabular}


general (D) es la media geométrica (multiplicativa) de todos las deseabilidades individuales $\left(d_{i}\right)$ en un rango de 0 (mínimo) a 1 (máximo) (Myers y Montgomery, 2002).

$$
D=\left(d_{1} \times d_{2} \times \ldots \times d_{n}\right)^{1 / n}=\left(\prod_{i=1}^{n} d_{i}\right)^{1 / n}
$$

Donde $n$ es el número de variables de respuesta que se optimizan simultáneamente. Si alguna de las respuestas queda fuera de su rango de conveniencia, la función global se convierte en cero. Para optimizaciones simultáneas cada variable de entrada debe tener un valor objetivo.

\section{Ejemplo}

El ejemplo que se presenta en este apartado es un modelo lineal con 7 variables, 5 de ellas de control y 2 de ruido, el modelo está estructurado de acuerdo con las características especificadas en la metodología y fue propuesto para realizar la experimentación. Se consideró un error de $\mathrm{N}(0,2)$, que indica una distribución normal con una media de 0 y una varianza de 2 , con el objetivo de simular un ruido natural en cada modelo. Los pasos para el planteamiento de este ejemplo se describen a continuación:

- Paso 1. Se seleccionó un modelo lineal que contenía 7 variables, 5 de ellas fueron de control (A, B, C, D, E) y 2 fueron de ruido ( $F$ y $G$ ).

- Paso 2. De acuerdo con la metodología, la mitad de las variables de control, A, B y C, se declararon significativas, al asignarles coeficientes de regresión que eran al menos tres veces la varianza del error. De la mima forma, la mitad de las variables de ruido se consideraron significativas, en este caso 1 variable, la variable G.

- Paso 3. Se determinaron las interacciones de control por control que estarían presentes en el modelo, iniciando con la mitad de las interacciones posibles, en este caso las posibilidades son AB, AC y BC. Tomando el criterio establecido en la metodología de redondear hacia el entero superior próximo, se consideraron significativas 2 interacciones $\mathrm{AB}$ y $\mathrm{BC}$. De la misma forma se establecieron las interacciones de control por ruido, en este caso las posibles interacciones son AG, BG y CG, según el mismo criterio de redondeo se tomaron como significativas las interacciones $A G$ y $C G$.
- Paso 4. Se definió el modelo verdadero como:

$$
\begin{aligned}
Y= & 50+4 A+7 B+16 C+5 G-6 A B-6 B C \\
& +2 A G+5 C G+N(0,2)
\end{aligned}
$$

Se simularon dos conjuntos de información utilizando simulación Monte Carlo y se realizaron dos análisis independientes utilizando las técnicas de Taguchi y el enfoque tradicional, note que los dos conjuntos de información generados se derivaron del mismo modelo verdadero.

- Paso 5. Una vez analizado el modelo con los dos enfoques de experimentación robusta y al no encontrarse diferencias significativas entre los resultados proporcionados por los dos métodos, se procede conforme a la metodología establecida a incrementar el número de variables de control e interacciones de control por control, partiendo de las mismas características iniciales donde el modelo lineal que contiene 7 variables, 5 de las cuales son de control (A, B, C, D, E) y 2 son variables de ruido (F y G).

- Paso 6. Las variables de control significativas en el modelo se incrementaron de 3 que eran, A, B y C, a 4 que fueron A, B, C y D.

- Paso 7. Las posibles interacciones de control por control se incrementaron a seis, AB, AC, AD, BC, BD y $\mathrm{CD}$; por lo tanto se declararon tres de ellas significativas, AB, BC y CD. En cuanto a las interacciones de control por ruido, estas no se incrementaron como previamente se indicó en la metodología, por tanto son 2 y corresponden a AG y CG, es así como queda establecido el siguiente modelo.

$$
\begin{aligned}
Y= & 50+4 A+7 B+16 C-9 D+5 G-6 A B \\
& -6 B C+6 C D+2 A G+5 C G+N(0,2)
\end{aligned}
$$

- Paso 8. Se procede al análisis y esta vez sí se detectaron diferencias significativas entre los resultados proporcionados por los dos enfoques de diseño experimental robusto.

- Paso 9. Después del análisis, se continúa aumentando la cantidad y la significancia de las interacciones de control por control para generar modelos saturados.

La siguiente sección muestra los conjuntos de información simulados para los enfoques de Taguchi y tradicional derivados del modelo verdadero (4), los conjuntos de información simulada para el modelo verdadero (3) y (5) no incluidos aquí, se generarían de la misma manera utilizando simulación Monte Carlo. 


\section{Enfoque de Taguchi}

La información simulada a partir del modelo verdadero (4) se muestra en la figura 3, esta información se analizó con el software de diseño experimental Qualitek-4 y Minitab simultáneamente. Para esta metodología se construye inicialmente un arreglo cruzado de Taguchi (L8 en este caso) (Castaño y Domínguez, 2010). A continuación se transforma el arreglo cruzado en su forma estándar, es decir, los factores de ruido quedan paralelos a los factores de control; esto se hace con el objeto de facilitar el análisis y la comparación de resultados sin alterar la esencia del enfoque experimental.

\section{Enfoque tradicional}

Se generó de la misma manera a partir del modelo verdadero (4), el diseño utilizado corresponde al $2^{7-1}$ de la tabla de diseños de resolución mixta de Lucas y Borkowski (1997), el cual recibe el nombre de diseño 7C. El diseño 7C contiene 7 variables, de las cuales hasta 5 pueden ser de control y 2 de ruido, es un diseño fraccionado $2^{7-1}$ de resolución VII. La tabla 2 muestra un extracto del conjunto de información generado.

El objetivo principal de esta investigación es marcar las diferencias existentes entre ambas metodologías en lo que respecta a la correcta identificación de las interacciones de control por control, además de medir otros indicadores para ambos métodos. Algunos de los indi- cadores de desempeño pueden obtenerse directamente del software, sin embargo hay otros que se tuvieron que calcular a partir de la información simulada, tal es el caso del estadístico AMSEE que requiere información de la media, así como el resultado de un producto vectorial de las diferencia de las betas del modelo verdadero y las betas del modelo del análisis de regresión.

\section{Comparación de estadísticos}

Esta investigación se enfocó en comparar dos metodologías de diseño experimental robusto, para lo cual fueron sometidos a análisis alrededor de 96 experimentos simulados, fueron incluidos múltiples diseños experimentales, desde diseños $2^{4}$ hasta $2^{10}$.

Se trabajó con 2 tipos de variantes, la primera fue la alteración de los valores de las betas en las interacciones de control por control, incrementándolas en orden ascendente y en múltiplos de 3 y 6 respecto al error introducido en la simulación, con el fin de aumentar su nivel de significancia. El segundo cambio que se realizó en los modelos verdaderos fue aumentar el número de interacciones de control por control significativas, con el fin de saturar los modelos y cuantificar en qué punto alguno de los dos métodos dejaba de estimar correctamente estas interacciones.

La tabla 3 muestra los resultados para este ejemplo, se proporciona la siguiente información: se pueden observar los términos que fueron estimados en el modelo

\begin{tabular}{|c|c|c|c|c|c|c|c|}
\hline \multicolumn{8}{|c|}{ Arreglo cruzado de Taguchi } \\
\hline \multicolumn{8}{|c|}{ Arreglo Interno } \\
\hline Corrida & A & B & $\mathrm{C}$ & $\mathrm{AB}$ & $\begin{array}{l}\mathrm{E}= \\
\mathrm{AC}\end{array}$ & $\begin{array}{l}\mathrm{F}= \\
\mathrm{BC}\end{array}$ & $\begin{array}{c}\mathrm{D}= \\
\mathrm{ABC}\end{array}$ \\
\hline def & -1 & -1 & -1 & 1 & 1 & 1 & -1 \\
\hline afg & 1 & -1 & -1 & -1 & -1 & 1 & 1 \\
\hline beg & -1 & 1 & -1 & -1 & 1 & -1 & 1 \\
\hline ahd & 1 & 1 & -1 & 1 & -1 & -1 & -1 \\
\hline$c d g$ & -1 & -1 & 1 & 1 & -1 & -1 & 1 \\
\hline ace & 1 & -1 & 1 & -1 & 1 & -1 & -1 \\
\hline bef & -1 & 1 & 1 & -1 & -1 & 1 & -1 \\
\hline abcdefg & 1 & 1 & 1 & 1 & 1 & 1 & 1 \\
\hline
\end{tabular}
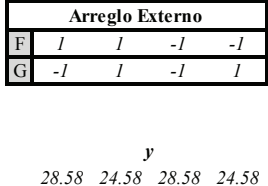

$\begin{array}{llll}4.23 & 28.23 & 4.23 & 28.23\end{array}$

$\begin{array}{llll}26.5 & 42.5 & 26.5 & 42.5\end{array}$

$\begin{array}{lllll}58.64 & 62.64 & 58.64 & 62.64\end{array}$

$\begin{array}{llll}43.98 & 59.98 & 43.98 & 59.98\end{array}$

$\begin{array}{llll}76.34 & 80.34 & 76.34 & 80.34\end{array}$

$\begin{array}{llll}71.24 & 67.24 & 71.24 & 67.24 \\ 50.49 & 74.49 & 50.49 & 74.49\end{array}$ $\begin{array}{llll}50.49 & 74.49 & 50.49 & 74.49\end{array}$

Figura 3. Resultados de la simulación de información del enfoque de Taguchi en su forma cruzada y estándar

\begin{tabular}{|c|c|c|c|c|c|c|c|c|c|c|c|}
\hline \multicolumn{10}{|c|}{ Arreglo en forma estándar } \\
\hline Corrida & $\mathrm{A}$ & $\mathrm{B}$ & $\mathrm{C}$ & $\mathrm{AB}$ & $\mathrm{E}=\mathrm{AC}$ & $\mathrm{F}=\mathrm{BC}$ & $\mathrm{D}=\mathrm{ABC}$ & $\mathrm{F}$ & $\mathrm{G}$ & $\boldsymbol{y}$ \\
\hline 1 & -1 & -1 & -1 & 1 & 1 & 1 & -1 & 1 & -1 & 28.58 \\
2 & 1 & -1 & -1 & -1 & -1 & 1 & 1 & 1 & -1 & 4.23 \\
3 & -1 & 1 & -1 & -1 & 1 & -1 & 1 & 1 & -1 & 26.5 \\
4 & 1 & 1 & -1 & 1 & -1 & -1 & -1 & 1 & -1 & 58.64 \\
5 & -1 & -1 & 1 & 1 & -1 & -1 & 1 & 1 & -1 & 43.98 \\
6 & 1 & -1 & 1 & -1 & 1 & -1 & -1 & 1 & -1 & 76.34 \\
7 & -1 & 1 & 1 & -1 & -1 & 1 & -1 & 1 & -1 & 71.24 \\
8 & 1 & 1 & 1 & 1 & 1 & 1 & 1 & 1 & -1 & 50.49 \\
9 & -1 & -1 & -1 & 1 & 1 & 1 & -1 & 1 & 1 & 24.58 \\
10 & 1 & -1 & -1 & -1 & -1 & 1 & 1 & 1 & 1 & 28.23 \\
11 & -1 & 1 & -1 & -1 & 1 & -1 & 1 & 1 & 1 & 42.5 \\
12 & 1 & 1 & -1 & 1 & -1 & -1 & -1 & 1 & 1 & 62.64 \\
13 & -1 & -1 & 1 & 1 & -1 & -1 & 1 & 1 & 1 & 59.98 \\
14 & 1 & -1 & 1 & -1 & 1 & -1 & -1 & 1 & 1 & 80.34 \\
15 & -1 & 1 & 1 & -1 & -1 & 1 & -1 & 1 & 1 & 67.24 \\
16 & 1 & 1 & 1 & 1 & 1 & 1 & 1 & 1 & 1 & 74.49 \\
17 & -1 & -1 & -1 & 1 & 1 & 1 & -1 & -1 & -1 & 28.58 \\
18 & 1 & -1 & -1 & -1 & -1 & 1 & 1 & -1 & -1 & 4.23 \\
19 & -1 & 1 & -1 & -1 & 1 & -1 & 1 & -1 & -1 & 26.5 \\
20 & 1 & 1 & -1 & 1 & -1 & -1 & -1 & -1 & -1 & 58.64 \\
21 & -1 & -1 & 1 & 1 & -1 & -1 & 1 & -1 & -1 & 43.98 \\
22 & 1 & -1 & 1 & -1 & 1 & -1 & -1 & -1 & -1 & 76.34 \\
23 & -1 & 1 & 1 & -1 & -1 & 1 & -1 & -1 & -1 & 71.24 \\
24 & 1 & 1 & 1 & 1 & 1 & 1 & 1 & -1 & -1 & 50.49 \\
25 & -1 & -1 & -1 & 1 & 1 & 1 & -1 & -1 & 1 & 24.58 \\
26 & 1 & -1 & -1 & -1 & -1 & 1 & 1 & -1 & 1 & 28.23 \\
27 & -1 & 1 & -1 & -1 & 1 & -1 & 1 & -1 & 1 & 42.5 \\
28 & 1 & 1 & -1 & 1 & -1 & -1 & -1 & -1 & 1 & 62.64 \\
29 & -1 & -1 & 1 & 1 & -1 & -1 & 1 & -1 & 1 & 59.98 \\
30 & 1 & -1 & 1 & -1 & 1 & -1 & -1 & -1 & 1 & 80.34 \\
31 & -1 & 1 & 1 & -1 & -1 & 1 & -1 & -1 & 1 & 67.24 \\
32 & 1 & 1 & 1 & 1 & 1 & 1 & 1 & -1 & 1 & 74.49 \\
\hline
\end{tabular}


de regresión lineal, también se muestran los valores para $R^{2} R_{\text {aj }}^{2}$ y $R_{\text {pred }}^{2}$ y los errores tipo I y II. Los errores tipo I y tipo II indican si el modelo reporto términos adicionales (tipo I) o si no fue capaz de estimar un término contenido en el modelo verdadero (tipo II). Se pueden observar a detalle qué términos del modelo verdadero fueron estimados en el análisis de regresión, además en cursiva se muestran las interacciones de control por control que cada metodología consideró significativas. Los porcentajes finales muestran $1 \%$ de términos que fueron estimados correctamente para cada modelo, de la misma forma se presenta el porcentaje de interacciones de control por control significativas que fueron estimadas correctamente, las cuales son las de mayor importancia para el presente estudio.

La columna marcada como: "sin interacciones de control por control", muestra los experimentos en la que los diseños solo cumplían la característica de ser robustos, es decir, solo contenían interacciones de control por ruido. En la sección "con interacciones de control por control significativas", se muestra la parte de la experimentación, donde se agregaron como se indicó en la metodología, 50\% de interacciones de control por control como significativas. Finalmente en la columna "con mayor número de interacciones de control por control altamente significativas", se muestran los diseños que fueron saturados con interacciones de control por control tanto en número de interacciones, como en nivel de significancia de estas interacciones.

\section{Búsqueda de valores que optimizan la respuesta}

El modelo (4) se analizó para detectar si el enfoque de Taguchi era capaz de estimar todas las interacciones de

Tabla 2. Resultados de la simulación de información, método tradicional

Método tradicional para un diseño 7C

Modelo verdadero $Y=50+4 A+7 B+16 C-9 D+5 G-6 A B-6 B C+6 C D+2 A G+5 C G+N(0,2)$

\begin{tabular}{crrrrrrrc}
\hline Corrida & A & B & C & D & E & F & G & y \\
1 & -1 & -1 & -1 & -1 & -1 & -1 & 1 & 24.07 \\
2 & 1 & -1 & -1 & -1 & -1 & -1 & -1 & 43.77 \\
3 & -1 & 1 & -1 & -1 & -1 & -1 & -1 & 66.59 \\
$\ldots$ & $\ldots$ & $\ldots$ & $\ldots$ & $\ldots$ & $\ldots$ & $\ldots$ & $\ldots$ & $\ldots$ \\
63 & -1 & 1 & 1 & 1 & 1 & 1 & -1 & 56.96 \\
64 & 1 & 1 & 1 & 1 & 1 & 1 & 1 & 73.91 \\
\hline
\end{tabular}

Tabla 3. Resultados de simulación diseños $2^{7}$

\begin{tabular}{|c|c|c|c|c|c|c|c|c|c|c|}
\hline \multirow{2}{*}{$\begin{array}{l}\text { Modelo } \\
\text { Verdadero }\end{array}$} & \multicolumn{3}{|c|}{$\begin{array}{l}\text { Sin interacciones de control por } \\
\text { control }\end{array}$} & \multicolumn{4}{|c|}{$\begin{array}{l}\text { Con interacciones de control por } \\
\text { control significativas }\end{array}$} & \multicolumn{3}{|c|}{$\begin{array}{l}\text { Con mayor número de interacciones de } \\
\text { control por control altamente significativas }\end{array}$} \\
\hline & \multicolumn{3}{|c|}{$\begin{array}{c}2^{7}(5,2) \quad Y=50+4 A+7 B+16 C \\
-9 D+5 G+2 A G+5 C G+N(0,2)\end{array}$} & \multicolumn{4}{|c|}{$\begin{array}{c}2^{7}(5,2) Y=50+4 A+7 B+16 C-9 D \\
+5 G-6 A B-6 B C+6 C D+2 A G \\
+5 C G+N(0,2)\end{array}$} & \multicolumn{3}{|c|}{$\begin{array}{c}2^{7}(5,2) Y=50+4 A+7 B+16 C-9 D-3 E \\
+5 G-6 A B+5 A E-6 B C+3 B E+6 C D \\
+2 A G+5 C G+N(0,1)\end{array}$} \\
\hline Indicadores & $\begin{array}{l}\text { Enfoque de } \\
\text { Taguchi }\end{array}$ & & $\begin{array}{l}\text { infoque } \\
\text { adicional }\end{array}$ & Enfoq & ue de Taguchi & & $\begin{array}{l}\text { Enfoque } \\
\text { adicional }\end{array}$ & Enfoque de Taguchi & Enfo & que tradicional \\
\hline $\begin{array}{l}\text { Términos } \\
\text { estimados en } \\
\text { el modelo de } \\
\text { regresión }\end{array}$ & $\begin{array}{l}\text { A, B, C, D, G, } \\
\text { AG,CG }\end{array}$ & & $\begin{array}{l}\text { B, C, D, G, } \\
A G, C G\end{array}$ & & $\begin{array}{l}\mathrm{B}, \mathrm{C}, \mathrm{D}, \mathrm{G} \\
\mathrm{AG}, \mathrm{CG}\end{array}$ & $\begin{array}{l}\text { A, B, } \\
\mathrm{BC}, \mathrm{C}\end{array}$ & $\begin{array}{l}\mathrm{C}, \mathrm{D}, \mathrm{G}, \mathrm{AB} \\
\mathrm{CD}, \mathrm{AG}, \mathrm{CG}\end{array}$ & $\begin{array}{l}\text { A, B, C, D, E, G, } \\
\text { BC, AG, CG }\end{array}$ & $\begin{array}{r}\mathrm{A}, \mathrm{B}, \\
\mathrm{BC},\end{array}$ & $\begin{array}{l}\text { D, E, AB, AE, } \\
\text { E CD, AG,CG }\end{array}$ \\
\hline$R^{2} / R_{a j}^{2} / R_{p r e d}^{2}(\%)$ & $99 \quad 98.7$ & 99.2 & $99.1 \quad 99$ & 99.5 & 98.1 & 99.2 & 98.6 & 99.299 .1 & 99.8 & 99.7 \\
\hline $\begin{array}{l}\text { Error tipo I } \\
\text { (Modelo) }\end{array}$ & No & & No & & No & & No & No & & No \\
\hline $\begin{array}{l}\text { Error tipo II } \\
\text { (Modelo) }\end{array}$ & No & & No & & $\mathrm{Si}$ & & No & $\mathrm{Si}$ & & No \\
\hline No error & $\mathrm{Si}$ & & $\mathrm{Si}$ & & No & & $\mathrm{Si}$ & No & & $\mathrm{Si}$ \\
\hline $\begin{array}{l}\text { Número de } \\
\text { corridas }\end{array}$ & 32 & & 64 & & 32 & & 64 & 32 & & 64 \\
\hline $\begin{array}{l}\text { \% términos } \\
\text { estimados } \\
\text { correctamente }\end{array}$ & $100 \%$ & & $100 \%$ & & $80.0 \%$ & & $100 \%$ & $69 \%$ & & $100 \%$ \\
\hline $\begin{array}{l}\% \text { interacciones } \\
\text { de } C^{*} \mathrm{C} \\
\text { estimadas }\end{array}$ & $100 \%$ & & $100 \%$ & & $33.3 \%$ & & $100 \%$ & $20 \%$ & & $100 \%$ \\
\hline
\end{tabular}


control por control, al detectarse que no, el modelo se optimizó para comprobar si esta situación repercute en los resultados esperados para la variable de respuesta.

$$
\begin{aligned}
Y= & 50+4 A+7 B+16 C-9 D+5 G-6 A B \\
& -6 B C+6 C D+2 A G+5 C G+N(0,2)
\end{aligned}
$$

El procedimiento que se siguió para optimizar las variables de respuesta para los conjuntos de información generados bajo el enfoque de Taguchi fue utilizar arreglos cruzados y mediante ayuda del software Qualitek-4, se lograron obtener las condiciones de operación óptimas. Observe que este modelo corresponde a un 27 que contiene 5 factores de control y 2 de ruido, por lo que es necesario utilizar un arreglo L8 (arreglo interno) y un L4 (arreglo externo). El objetivo es maximizar la respuesta, es decir, un mayor es mejor; el proceso de optimización al utilizar el software es automático. Las condiciones de operación óptimas obtenidas se muestran en la tabla 4, note que el software recomienda colocar las variables A, B y C a nivel alto y las variables, E y $\mathrm{D}$, a nivel bajo.

Para el enfoque tradicional se trabajó también el mismo modelo (4) pero esta vez se utilizó la función de deseabilidad para realizar el proceso de optimización. Se generó un diseño $2^{7-1}$ de la tabla de Lucas y Borkowski (1997), el cual contiene 7 factores (5 factores de control y 2 factores de ruido). El factor de deseabilidad varía de 0 a 1 , y es 1 el que logra optimizar la respuesta. La tabla 5 muestra los resultados obtenidos, note que las condiciones de operación óptimas toman valores de -1 a 1, en forma adicional $\mathrm{E}$ se reporta como no significativo.
Una vez que se obtuvieron los niveles recomendados para los factores de control que maximizaban la variable respuesta para cada enfoque de diseño experimental, se simularon 300,000 respuestas para cada enfoque de la siguiente manera: los niveles de los factores de control recomendados para cada enfoque se sustituyeron en la ecuación (4) y los niveles para los factores de ruido y el error se variaron en forma aleatoria durante la simulación, para emular un proceso real, lo que dio lugar a dos histogramas diferentes que representan la distribución de la variable de respuesta dentro de cada enfoque. Estos histogramas se analizaron para conocer los niveles de variabilidad, media y desviación estándar generados por cada metodología. De la misma manera se determinó qué metodología optimizó de forma más eficiente la variable de respuesta. La figura 4 presenta los histogramas generados.

Al optimizarse ambos modelos por los dos distintos enfoques, se encuentran diferencias significativas, la media del método tradicional fue $12 \%$ mayor con respecto a la obtenida mediante el enfoque de Taguchi. Dado que el objetivo era maximizar se concluye que es más conveniente utilizar el método tradicional, el cual a su vez mantiene una variabilidad similar al enfoque de Taguchi. En la siguiente sección se muestran a detalle los resultados de todos los experimentos y modelos comparados.

\section{Discusión y análisis de resultados}

El ejemplo mostrado es solo uno de varios modelos analizados. El total de resultados para cada diseño respecto a la estimación porcentual se muestra con detalle

Tabla 4. Valores operacionales óptimos proporcionados por Qualitek-4

\begin{tabular}{cc}
\hline $\mathrm{Y}=50+4 \mathrm{~A}+7 \mathrm{~B}+16 \mathrm{C}-9 \mathrm{D}+5 \mathrm{G}-6 \mathrm{AB}-6 \mathrm{BC}+6 \mathrm{CD}+2 \mathrm{AG}+5 \mathrm{CG}+\mathrm{N}(0,2)$ \\
\hline Factor & Nivel Recomendado \\
\hline $\mathrm{A}$ & 2 \\
$\mathrm{~B}$ & 2 \\
$\mathrm{C}$ & 2 \\
$\mathrm{D}$ & 1 \\
$\mathrm{E}$ & 1 \\
\hline
\end{tabular}

\begin{tabular}{|c|c|c|c|c|c|c|c|c|}
\hline \multirow{3}{*}{$\begin{array}{c}\text { Soluciones } \\
\text { Número } \\
1\end{array}$} & \multicolumn{8}{|c|}{$Y=50+4 A+7 B+16 C-9 D+5 G-6 A B-6 B C+6 C D+2 A G+5 C G+N(0,2)$} \\
\hline & \multirow{2}{*}{$\frac{\mathrm{A}}{0.98}$} & \multirow{2}{*}{$\frac{B}{-0.84}$} & \multirow{2}{*}{$\frac{C}{0.99}$} & \multirow{2}{*}{$\frac{\mathrm{D}}{-1.00}$} & \multirow{2}{*}{$\frac{E^{*}}{0.92}$} & \multirow{2}{*}{$\frac{\text { Respuesta }}{76.71}$} & \multicolumn{2}{|c|}{ Deseabilidad } \\
\hline & & & & & & & 0.95 & Seleccionado \\
\hline 2 & 0.98 & -0.84 & 0.99 & -1.00 & -0.92 & 76.71 & 0.95 & \\
\hline 3 & 0.95 & -0.80 & 1.00 & -0.88 & -0.91 & 76.10 & 0.95 & \\
\hline
\end{tabular}

Tabla 5. Optimización en software Design expert 
en la tabla 6. Se utilizó la siguiente simbología: $2^{n}(x, z)$, donde $n$ indica el número total de factores que contiene el diseño, estos varían de 5 a 10, $x$ indica el número de variables de control que contiene el modelo y, finalmente, $z$ indica el número de variables de ruido dentro del modelo.

Como se puede observar en la tabla 6, al comparar los resultados de ambas metodologías, se nota una diferencia porcentual bastante significativa en la capacidad para estimar interacciones de control por control por parte del enfoque de Taguchi. Esta tendencia de reducción de la capacidad para estimar estas las interacciones se incrementa cuando los modelos simulados se saturan con interacciones de control por control.

Otros estadísticos utilizados en este estudio, nombrados como indicadores de desempeño, fueron $\mathrm{R}^{2}$, error tipo I, error tipo II, no error y el número de corridas; en la tabla 7 se presenta el resumen de resultados de todos los experimentos realizados. La fila de \% promedio ubicada al final de la tabla representa el prome-

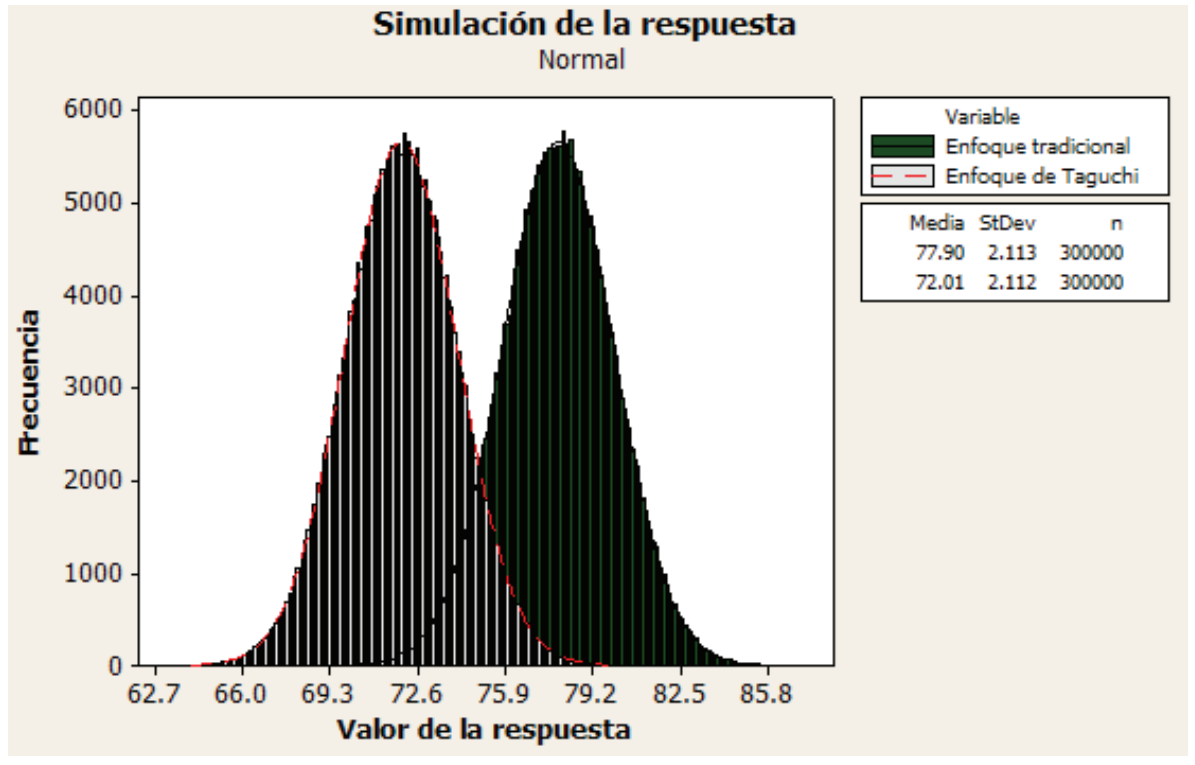

Figura 4. Comparación de la variable de respuesta simulada

Tabla 6. Porcentaje de estimación de términos e interacciones de control por control

\begin{tabular}{|c|c|c|c|c|c|c|c|c|}
\hline \multirow{3}{*}{ Diseño } & \multicolumn{4}{|c|}{ Interacciones de control por control } & \multicolumn{4}{|c|}{ Términos estimados correctamente } \\
\hline & \multicolumn{2}{|c|}{$\begin{array}{l}\text { Con interacciones de control } \\
\text { por control significativas }\end{array}$} & \multicolumn{2}{|c|}{$\begin{array}{l}\text { Con mayor número de } \\
\text { interacciones de control } \\
\text { por control altamente } \\
\text { significativas }\end{array}$} & \multicolumn{2}{|c|}{$\begin{array}{l}\text { Con interacciones de control } \\
\text { por control significativas }\end{array}$} & \multicolumn{2}{|c|}{$\begin{array}{c}\text { Con mayor número de } \\
\text { interacciones de control } \\
\text { por control altamente } \\
\text { significativas }\end{array}$} \\
\hline & $\begin{array}{l}\text { Enfoque de } \\
\text { Taguchi }\end{array}$ & $\begin{array}{l}\text { Enfoque } \\
\text { Tradicional }\end{array}$ & $\begin{array}{l}\text { Enfoque de } \\
\text { Taguchi }\end{array}$ & $\begin{array}{l}\text { Enfoque } \\
\text { Tradicional }\end{array}$ & $\begin{array}{l}\text { Enfoque de } \\
\text { Taguchi }\end{array}$ & $\begin{array}{l}\text { Enfoque } \\
\text { Tradicional }\end{array}$ & $\begin{array}{l}\text { Enfoque de } \\
\text { Taguchi }\end{array}$ & $\begin{array}{c}\text { Enfoque } \\
\text { Tradicional }\end{array}$ \\
\hline $2^{5(3,2)}$ & $100.0 \%$ & $100.0 \%$ & $66.0 \%$ & $100.0 \%$ & $100.0 \%$ & $100.0 \%$ & $85.7 \%$ & $100.0 \%$ \\
\hline $2^{6(4,2)}$ & $33.3 \%$ & $100.0 \%$ & $40.0 \%$ & $100.0 \%$ & $77.8 \%$ & $100.0 \%$ & $75.0 \%$ & $100.0 \%$ \\
\hline $2^{7(4,3)}$ & $25.0 \%$ & $100.0 \%$ & $16.7 \%$ & $100.0 \%$ & $72.7 \%$ & $100.0 \%$ & $61.5 \%$ & $100.0 \%$ \\
\hline $2^{7(5,2)}$ & $33.0 \%$ & $100.0 \%$ & $20.0 \%$ & $100.0 \%$ & $80.0 \%$ & $100.0 \%$ & $69.2 \%$ & $100.0 \%$ \\
\hline $2^{8(5,3)}$ & $50.0 \%$ & $100.0 \%$ & $33.0 \%$ & $100.0 \%$ & $83.3 \%$ & $100.0 \%$ & $71.4 \%$ & $100.0 \%$ \\
\hline $2^{8(6,2)}$ & $50.0 \%$ & $100.0 \%$ & $20.0 \%$ & $100.0 \%$ & $84.6 \%$ & $100.0 \%$ & $66.7 \%$ & $100.0 \%$ \\
\hline $2^{9(6,3)}$ & $50.0 \%$ & $100.0 \%$ & $16.7 \%$ & $100.0 \%$ & $83.3 \%$ & $100.0 \%$ & $66.7 \%$ & $100.0 \%$ \\
\hline $2^{9(7,2)}$ & $20.0 \%$ & $100.0 \%$ & $14.3 \%$ & $100.0 \%$ & $69.2 \%$ & $100.0 \%$ & $62.5 \%$ & $100.0 \%$ \\
\hline $2^{10(6,4)}$ & $20.0 \%$ & $100.0 \%$ & $14.3 \%$ & $100.0 \%$ & $64.3 \%$ & $100.0 \%$ & $62.5 \%$ & $100.0 \%$ \\
\hline $2^{10(7,3)}$ & $20.0 \%$ & $100.0 \%$ & $14.3 \%$ & $100.0 \%$ & $57.1 \%$ & $100.0 \%$ & $62.5 \%$ & $100.0 \%$ \\
\hline $2^{10(8,2)}$ & $30.0 \%$ & $100.0 \%$ & $20.0 \%$ & $100.0 \%$ & $62.5 \%$ & $100.0 \%$ & $47.4 \%$ & $100.0 \%$ \\
\hline
\end{tabular}


dio general de la $\mathrm{R}^{2}$ de todos los experimentos. Para los errores I y II y no error representa el porcentaje en el que el estadístico dio resultado positivo en el experimento, es decir cuando se presentó. Respecto al número de corridas es una media general de las corridas que utilizaba cada enfoque de experimentación robusta.

Uno de los objetivos de este estudio fue la búsqueda de valores operacionales que optimicen la respuesta para ambos enfoques, para verificar si el hecho de estimar o no las interacciones de control por control tiene un efecto significativo en los parámetros recomendados por cada metodología, se optimizó cada metodología de acuerdo con su procedimiento típico de búsqueda, el índice de señal a ruido para los arreglos de Taguchi y la función de deseabilidad para los arreglos del enfoque tradicional. Se consideró tanto maximización como minimización.

Modelos específicos que contenían interacciones de control por control fueron seleccionados, estos modelos pudieron dar una respuesta muy clara de las diferencias entre ambos enfoques al momento de optimizar las respuestas. Los procesos de optimización se realizaron para cada uno de los modelos: $2^{4}, 2^{5} \ldots$ hasta el $2^{10}$. El conjunto de resultados obtenidos se muestran en la tabla 8; esta tabla muestra el resumen de las optimizaciones con la media y la desviación estándar de las 300,000 variables de respuesta simuladas para cada modelo dentro de cada enfoque y para cada objetivo, maximización o minimización.

Con los datos obtenidos de todas las optimizaciones, se observó que el enfoque tradicional logra una mejor optimización tanto para maximización como para minimización con respecto al enfoque de Taguchi. Si consideramos que el método tradicional llega a $100 \%$ y tomando esto como base, la optimización por el enfoque de Taguchi llega en promedio a $63 \%$ para maximización y a $56 \%$ para minimización. Un ejemplo claro es el diseño $2^{9}$, que se muestra en la tabla 7 , si el objetivo es maximizar la respuesta, la técnica de Taguchi obtiene una media de 5, mientras que el enfoque tradicional logra llegar a 48.6, una diferencia claramente significativa.

\section{Conclusiones}

Una de las principales desventajas de trabajar con el diseño experimental robusto de Taguchi es que no es capaz de estimar interacciones de control por control, en forma adicional, los experimentadores no cuentan con información a priori sobre la presencia y nivel de significancia de estas interacciones y la literatura actual únicamente proporciona información cualitativa y no cuantitativa sobre las desventajas de los métodos de Taguchi. Por tales motivos, es de interés analizar y cuantificar las diferencias existentes entre los resultados proporcionados por el enfoque de Taguchi y el enfoque tradicional en presencia de interacciones de control por control, con esas diferencias expresadas en términos de estadísticos y porcentajes.

El presente trabajo de investigación realizó una comparación directa entre los enfoques de Taguchi y tradicional; para lograr esto, los diseños de Taguchi se colocaron en forma estándar y se analizaron con software para determinar sus propiedades, mismas que fue-

Tabla 7. Resumen global de Indicadores de desempeño

\begin{tabular}{|c|c|c|c|c|c|c|c|c|c|c|}
\hline \multirow[b]{3}{*}{ Diseño } & \multicolumn{10}{|c|}{ Indicadores de desempeño } \\
\hline & \multicolumn{2}{|r|}{ R2 } & \multicolumn{2}{|c|}{ Error tipo I } & \multicolumn{2}{|c|}{ Error tipo II } & \multicolumn{2}{|c|}{ No error } & \multicolumn{2}{|c|}{ Número de corridas } \\
\hline & $\begin{array}{l}\text { Enfoque } \\
\text { de } \\
\text { Taguchi }\end{array}$ & $\begin{array}{l}\text { Enfoque } \\
\text { Tradicional }\end{array}$ & $\begin{array}{c}\text { Enfoque } \\
\text { de Taguchi }\end{array}$ & $\begin{array}{l}\text { Enfoque } \\
\text { Tradicional }\end{array}$ & $\begin{array}{c}\text { Enfoque } \\
\text { de } \\
\text { Taguchi }\end{array}$ & $\begin{array}{l}\text { Enfoque } \\
\text { Tradicional }\end{array}$ & $\begin{array}{l}\text { Enfoque } \\
\text { de } \\
\text { Taguchi }\end{array}$ & $\begin{array}{l}\text { Enfoque } \\
\text { Tradicional }\end{array}$ & $\begin{array}{c}\text { Enfoque } \\
\text { de } \\
\text { Taguchi }\end{array}$ & $\begin{array}{l}\text { Enfoque } \\
\text { Tradicional }\end{array}$ \\
\hline $2^{5(3,2)}$ & $99.5 \%$ & $99.8 \%$ & No & No & No & No & $\mathrm{Si}$ & $\mathrm{Si}$ & 32 & 16 \\
\hline $2^{6(4,2)}$ & $91.8 \%$ & $97.9 \%$ & $\mathrm{Si}$ & No & $\mathrm{Si}$ & No & No & $\mathrm{Si}$ & 32 & 32 \\
\hline $2^{7(4,3)}$ & $99.5 \%$ & $99.5 \%$ & $\mathrm{Si}$ & No & $\mathrm{Si}$ & No & No & $\mathrm{Si}$ & 32 & 32 \\
\hline $2^{7(5,2)}$ & $99.5 \%$ & $99.2 \%$ & No & No & $\mathrm{Si}$ & No & No & $\mathrm{Si}$ & 32 & 64 \\
\hline $2^{8(5,3)}$ & $99.8 \%$ & $99.6 \%$ & No & No & $\mathrm{Si}$ & No & No & $\mathrm{Si}$ & 32 & 64 \\
\hline $2^{8(6,2)}$ & $99.4 \%$ & $99.4 \%$ & $\mathrm{Si}$ & No & $\mathrm{Si}$ & No & No & $\mathrm{Si}$ & 32 & 64 \\
\hline $2^{9(6,3)}$ & $99.6 \%$ & $99.4 \%$ & No & No & $\mathrm{Si}$ & No & No & $\mathrm{Si}$ & 32 & 64 \\
\hline $2^{9(7,2)}$ & $99.6 \%$ & $99.5 \%$ & $\mathrm{Si}$ & No & $\mathrm{Si}$ & No & No & $\mathrm{Si}$ & 32 & 128 \\
\hline $2^{10(6,4)}$ & $99.6 \%$ & $99.3 \%$ & No & No & $\mathrm{Si}$ & No & No & $\mathrm{Si}$ & 64 & 128 \\
\hline $2^{10(7,3)}$ & $99.3 \%$ & $99.2 \%$ & $\mathrm{Si}$ & No & $\mathrm{Si}$ & No & No & $\mathrm{Si}$ & 64 & 128 \\
\hline $2^{10(8,2)}$ & $99.2 \%$ & $99.4 \%$ & $\mathrm{Si}$ & No & $\mathrm{Si}$ & No & No & $\mathrm{Si}$ & 48 & 128 \\
\hline$\%$ promedio & $98.8 \%$ & $99.3 \%$ & $55 \%$ & $100 \%$ & $91 \%$ & $100 \%$ & $9 \%$ & $100 \%$ & 39 & 77 \\
\hline
\end{tabular}


Tabla 8. Optimización de modelos

\begin{tabular}{|c|c|c|c|c|c|}
\hline \multicolumn{2}{|c|}{ Enfoque } & \multicolumn{2}{|c|}{ Taguchi } & \multicolumn{2}{|c|}{ Tradicional } \\
\hline Diseño & Objetivo & Media (m) & Desviación estándar & Media (m) & Desviación estándar \\
\hline \multicolumn{2}{|c|}{ Modelo } & \multicolumn{4}{|c|}{$\mathrm{Y}=16+7 \mathrm{~A}-6 \mathrm{~B}+4 \mathrm{D}-9 \mathrm{AB}+4 \mathrm{BD}+\mathrm{N}(0,2)$} \\
\hline \multirow{2}{*}{$2^{5}$} & Maximizar & 8.001 & 0.9986 & 38 & 1.002 \\
\hline & Minimizar & 12.001 & 2.847 & 6 & 1.001 \\
\hline \multicolumn{2}{|c|}{ Modelo } & \multicolumn{4}{|c|}{$Y=50+7 B+6 C+5 D+E+2 B C+2 B D-10 C D-5 B E-3 D E+N(0,2)$} \\
\hline \multirow{2}{*}{$2^{6}$} & Maximizar & 62.001 & 2.03 & 68 & 2.026 \\
\hline & Minimizar & 46 & 2.23 & 32.12 & 2.24 \\
\hline \multicolumn{2}{|c|}{ Modelo } & \multicolumn{4}{|c|}{$\mathrm{Y}=50+4 \mathrm{~A}+7 \mathrm{~B}+16 \mathrm{C}-9 \mathrm{D}+5 \mathrm{G}-6 \mathrm{AB}-6 \mathrm{BC}+6 \mathrm{CD}+2 \mathrm{AG}+5 \mathrm{CG}+\mathrm{N}(0,2)$} \\
\hline \multirow{2}{*}{$2^{7}$} & Maximizar & 72.01 & 2.112 & 77.91 & 2.106 \\
\hline & Minimizar & 8.004 & 3.333 & 4.05 & 3.33 \\
\hline \multicolumn{2}{|c|}{ Modelo } & \multicolumn{4}{|c|}{$\begin{array}{c}\mathrm{Y}=50+9 \mathrm{~B}+10 \mathrm{C}-6 \mathrm{D}-9 \mathrm{E}+5 \mathrm{~F}+8 \mathrm{H}-12 \mathrm{BC}-2 \mathrm{BD}+2 \mathrm{CE}+2 \mathrm{EF}+\mathrm{BH} \\
+7 \mathrm{CH}+5 \mathrm{EH}+\mathrm{N}(0,2)\end{array}$} \\
\hline \multirow{2}{*}{$2^{8}$} & Maximizar & 75 & 4.182 & 81.62 & 2.118 \\
\hline & Minimizar & 20.99 & 2.69 & 11.5 & 3.01 \\
\hline \multicolumn{2}{|c|}{ Modelo } & \multicolumn{4}{|c|}{ 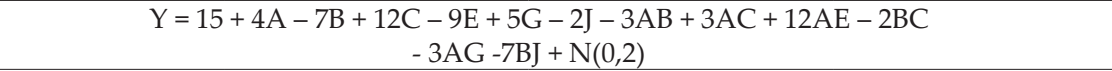 } \\
\hline \multirow{2}{*}{$2^{9}$} & Maximizar & 5 & 3.22 & 48.6 & 1.85 \\
\hline & Minimizar & -7 & 3.222 & -20 & 4.133 \\
\hline \multicolumn{2}{|c|}{ Modelo } & \multicolumn{4}{|c|}{$\begin{array}{c}\mathrm{Y}=40+10 \mathrm{~A}-6 \mathrm{~B}+7 \mathrm{C}+5 \mathrm{D}+8 \mathrm{E}-3 \mathrm{G}+5 \mathrm{~K}-3 \mathrm{AB}+3 \mathrm{AC}-4 \mathrm{AD}+12 \mathrm{BD} \\
+2 \mathrm{CG}+4 \mathrm{CE}-4 \mathrm{AK}+3 \mathrm{BK}+3 \mathrm{CK}+\mathrm{N}(0,4)\end{array}$} \\
\hline \multirow{2}{*}{$2^{10}$} & Maximizar & 69.01 & 4.014 & 89.33 & 4.019 \\
\hline & Minimizar & 3.002 & 5 & -3.65 & 4.92 \\
\hline
\end{tabular}

ron contrastadas contra las del enfoque tradicional, los estadísticos considerados incluyeron términos estimados en el modelo de regresión, $\mathrm{R}^{2}, \mathrm{R}_{\mathrm{aj}}^{2} \mathrm{R}_{\text {pred }}^{2}$, error tipo I, error tipo II, no error, número de corridas, \% de términos estimados correctamente y $\%$ de interacciones de control por control estimadas correctamente.

Al realizar el análisis de resultados de cada experimento, se concluye que la diferencia primordial entre los enfoques de Taguchi y tradicional es que los arreglos cruzados de Taguchi no tienen la capacidad para estimar todos los términos significativos en un modelo, esta situación empeora cuando existen interacciones de control por control significativas. En cuanto a la eficiencia, los diseños de Taguchi tienden a ser más económicos, ya que tienden a utilizar menos corridas por diseño. Sin embargo, de manera general los arreglos de Taguchi utilizan más corridas que el enfoque tradicional y la información que se obtiene siempre es menor. Las $\mathrm{R}^{2}, \mathrm{R}_{\text {aj, }}^{2} \mathrm{y} \mathrm{R}_{\text {pred }}^{2}$ toman valores similares en los dos enfoques, con una diferencia mínima en favor del enfoque tradicional.

En cuanto a los errores tipo I el enfoque de Taguchi estimo en $55 \%$ de los diseños, términos adicionales, mientras que el tradicional nunca confundió esta estimación; de especial interés es el error tipo II puesto que este evalúa cuándo se omiten factores o interacciones significativos, dicho error fue cometido en $91 \%$ de los experimentos con el enfoque de Taguchi y nunca se presentó con el enfoque tradicional. El indicador de desempeño "no error", que evalúa cuando un diseño estima correctamente todos sus términos e interacciones, se pre- sentó en $100 \%$ en el enfoque tradicional y solo en $9 \%$ en el enfoque Taguchi.

La segunda parte del estudio se enfocó en optimizar los modelos de Taguchi y del enfoque tradicional, al determinar los niveles de factores capaces de maximizar o minimizar la respuesta para cada enfoque. El resultado de las optimizaciones fue que en todos los casos el enfoque de Taguchi es menos eficiente que el enfoque tradicional tanto al minimizar como al maximizar cualquier modelo.

\section{Referencias}

Castaño-Tostado E. y Domínguez-Domínguez J. Diseño de experimentos y análisis en ciencia y tecnologia, Querétaro, UAQ, 2010.

Del Castillo E., Alvarez M.J., Ilzarbe L., Viles E. A New Design Criterion for Robust Parameter Experiments. Journal of Quality Technology, volumen 279, 2007.

George B. Signal-to-Noise Ratios, Performance Criteria, and Transformations. TECHNOMETRIC, volumen 30 (número 1),1988: 18.

George B., Soren B. y Conrad F. Una explicación y critica de las contribuciones de Taguchi a la Ingeniería de Calidad. Quality and Reliability Engineering International, 1988: 123-133.

Kunert J., Auer C., Erdbrügge M., Ewers R. An Experiment to Compare Taguchi's Product Array and the Combined Array. Journal of Quality Technology, 2007: 1-17.

Kutner M.H., Neter J., Wasserman W. Applied Linear Statistical Models, M.A, Irwin, 1990.

Lucas J.M. y Borkowski J.J. Designs of Mixed Resolution for Process Robustness Studies. Technometrics, 1997: 63-70. 
Miró-Quesada G. y Del-Castillo E. A Dual-Response Approach to the Multivariate Robust Parameter Design Problem. Technometrics, 2004: 176-187.

Misra H. Quarterfold: a Data-Driven Sequential Augmentation Stratagey for Resolution IV Designs. The Florida Agricultural and Mechanical Uuniversity College of Engineering, Florida, 2006.

Montgomery D. Design and Analysis of Experiments, Nueva York, LIMUSA, 2009.

Montgomery D.C. Experimental Design for Product and Process Design and Development. Journal of the Royal Statistical Society, 1999: 159-177.

Myers R.G. y Montgomery D.C. Robust Parameter Design and Process Robustness Studies, 2002.

Peace G.S. Taguchi Methods: a Hands-On Approach, Massachusetts, Addison-Weasley, 1993.

Rios-Lira A.J. Sequential Experimentation Schemes For Resolution III, Robust and Mixed-Level Designs, Florida, 2008.

Rios A.J., Diosdado P., Hernandez S. Sequential Experimentation Approach for Robust Designs, en: II International Congress of Engineering and Technology, 2009, pp. 13-19
Roy R.K. Design of Experiments Using The Taguchi Approach: 16 Steps To Product and Process Improvement, Nueva York, John Willey \& Sons, Inc, 2001.

\section{Este artículo se cita:}

\section{Citación estilo Chicago}

Arias-Nava, Elías Heriberto, Armando Javier Ríos-Lira, José Antonio Vázquez-López, Russell Pérez-González. Estudio comparativo entre los enfoques de diseño experimental robusto de Taguchi y tradicional en presencia de interacciones de control por control. Ingeniería Investigación y Tecnología, XVI, 01 (2015): 131-142.

\section{Citación estilo ISO 690}

Arias-Nava E.H., Ríos-Lira A.J., Vázquez-López J.A., PérezGonzález R. Estudio comparativo entre los enfoques de diseño experimental robusto de Taguchi y tradicional en presencia de interacciones de control por control. Ingeniería Investigación y Tecnología, volumen XVI (número 1), enero-marzo 2015: 131-142.

\section{Semblanza de los autores}

Elías Heriberto Arias-Nava. Es ingeniero industrial egresado del Instituto Tecnológico de Ciudad Guzmán. Obtuvo el grado de maestro en ingeniería industrial por el Instituto Tecnológico de Celaya. Su tesis y área de interés están enfocadas al diseño de experimentos.

Armando Javier Ríos-Lira. Es profesor investigador de tiempo completo en el Departamento de Ingeniería Industrial del Instituto Tecnológico de Celaya. Recibió la licenciatura en ingeniería industrial en el I.T.C. y su maestría en ciencias y doctorado, ambos en ingeniería industrial por la Universidad Estatal de Florida. Es miembro del Sistema Nacional de Investigadores (SNI). Sus áreas de concentración incluyen el diseño de experimentos, análisis de regresión y simulación.

José Antonio Vázquez-López. Es profesor investigador del Instituto Tecnológico de Celaya. Pertenece al Sistema Nacional de Investigadores y cuenta con el reconocimiento de perfil deseable del PROMEP. Es doctor en ciencia y tecnología con especialidad en ingeniería industrial por el CIATEC, A.C. Su área de investigación es el reconocimiento de patrones por inteligencia artificial

Russell Pérez-González. Es ingeniero industrial egresado del Instituto Tecnológico de Tuxtla. Obtuvo el grado de maestro en ingeniería industrial por el Instituto Tecnológico de Celaya. Su tesis y área de interés están enfocadas al diseño de experimentos. 